\title{
Effects of density gradients and fluctuations at the plasma edge on ECEI measurements at ASDEX Upgrade
}

B Vanovac ${ }^{1}$, E Wolfrum ${ }^{2}$, S S Denk ${ }^{2}$, F Mink M $^{2,4}$, F M Laggner ${ }^{3}$, G Birkenmeier ${ }^{2,4}, \mathrm{M}$ Willensdorfer ${ }^{2}, \mathbf{E ~ V i e z z e r ~}^{2,5}, \mathbf{M ~ H o e l z l}^{2}$, S J Freethy $^{2}$, M G Dunne ${ }^{2}$, A Lessig ${ }^{2}$, N C Luhmann Jr. ${ }^{6}$, the ASDEX Upgrade Team and the EUROfusion MST1 Team ${ }^{7}$

${ }^{1}$ DIFFER - Dutch Institute for Fundamental Energy Research, Eindhoven, the Netherlands

${ }^{2}$ Max-Planck-Institut für Plasmaphysik, 85748 Garching, Germany

${ }^{3}$ Institute of Applied Physics, TU Wien, Fusion@ÖAW, 1040 Vienna, Austria

${ }^{4}$ Physik-Department E28, Technische Universität München, 85748 Garching, Germany

${ }^{5}$ Department of Atomic, Molecular, and Nuclear Physics, University of Seville, Seville, Spain

${ }^{6}$ Department of Applied Science, University of California at Davis, Davis, CA 95616, USA

7 See author list of "H. Meyer et al 2017 Nucl. Fusion 57 102014"

E-mail: B.Vanovac@differ.nl

July 2017

Abstract. Electron Cyclotron Emission Imaging (ECEI) provides measurements of electron temperature $\left(T_{e}\right)$ and its fluctuations $\left(\delta T_{e}\right)$. However, when measuring at the plasma edge, in the steep gradient region, radiation transport effects must be taken into account. It is shown that due to these effects, the Scrape-Off Layer (SOL) region is not accessible to the ECEI measurements in steady state conditions and that the signal is dominated by the shine-through emission. Transient effects, such as filaments, can change the radiation transport locally, but cannot be distinguished from the shinethrough. Local density measurements are essential for the correct interpretation of the electron cyclotron emission, since the density fluctuations influence the temperature measurements at the plasma edge. As an example, a low frequency $8 \mathrm{kHz}$ mode, which causes $10 \%$ to $15 \%$ fluctuations in the signal level of the ECEI, is analysed. The same mode has been measured with the Lithium Beam Emission Spectroscopy (Li-BES) density diagnostic, and is very well correlated in time with high frequency magnetic fluctuations. With radiation transport modelling of the electron cyclotron radiation in the ECEI geometry, it is shown that the density contributes significantly to the radiation temperature $\left(T_{\text {rad }}\right)$ and the experimental observations have shown the amplitude modulation in both density and temperature measurements. The poloidal velocity of the low frequency mode measured by the ECEI is $3 \mathrm{~km} / \mathrm{s}$. The calculated velocity of the high frequency mode measured with the magnetic pick-up coils is about $25 \mathrm{~km} / \mathrm{s}$. Velocities are compared with the $E \times B$ background flow velocity and possible explanations for the origin of the low frequency mode are discussed. 


\section{Introduction}

H-mode plasmas[21] are the foreseen scenario for the ITER operation[14]. This type of scenario is characterized by steep gradients and the pedestal build-up at the edge of the plasma. Edge Localized Modes (ELMs) [26, 13] lead to periodic relaxations of the gradients including sudden losses of heat and particles into the unconfined region in $\sim$ 1-2 ms time scale. These periodic bursts can cause intolerable heat loads on divertor target plates and the erosion of plasma facing components. In order to maintain the high confinement of heat and particles, but with no impurity accumulation and without large uncontrollable ELMs, ELMs need to be mitigated. For such a full control the dynamics of ELMs and ELM associated phenomena should be better understood.

The Electron Cyclotron Emission Imaging (ECEI) diagnostic, with multiple lines of sight (LOS), where each LOS behaves like a conventional 1D radiometer, measures the radiation temperature and its fluctuations on $\mu$ s time scales and is specially suitable for $2 \mathrm{D}$ or quasi-3D visualisation of MHD phenomena. It can thus help provide more insight into the behaviour of the relative temperature fluctuations associated with the ELM cycle. To date, ECEI has been installed on many tokamaks including: ASDEX Upgrade[3], KSTAR[25], EAST[24], DIII-D[18] and HL-2A[9].

In the case of optically thick plasmas, where optical thickness is proportional to the product of electron density and temperature, the intensity of the cyclotron radiation equals the level of black body emission. In this case, under the condition that the electrons are in a local thermodynamic equilibrium, radiation temperature is equal to the electron temperature. However, when measuring at the plasma edge, where the density and temperatures are relatively low, the electron cyclotron emission no longer equals the black body radiation and the density contribution cannot be neglected. In this case the radiation transport equation is solved in order to obtain the radiation temperatures. It is important to note here that the geometry under which the light is collected plays a very important role. Under perpendicular observation of the plasma, where the line of sight is perpendicular to the magnetic field line, there is no significant parallel contribution of the cyclotron emission and the Doppler broadening can be neglected. In this case, the radial resolution of the system is set by the the band-pass filtering of the intermediate frequency (IF) signal around the central frequency in the second down-conversion process (the IF bandwidth). If, however, the observation angle is slightly oblique, as is the case with the present ECEI system on ASDEX Upgrade, this Doppler broadening must be taken into account. In this case the radial resolution of the system is set by both, IF broadening and Doppler broadening. Another important point is related to hot $\mathrm{H}-$ mode plasmas where the relativistically down-shifted emission contributes significantly to the low field side emission. This effect shifts the peak of the resonance towards the higher magnetic field[19]. When all the mentioned effects are taken into account, measurement positions in the case of oblique diagnostics can significantly differ from the cold resonances as shown in[6].

In this work, we examine a particular H-mode discharge during the phase between 
two subsequent ELM crashes, where the large amplitude density fluctuation alongside the temperature fluctuations are measured. The main question to answer in this work is if and how much of the fluctuating signal measured with ECEI, which is considered a temperature diagnostic, is affected by the variation of the density at the plasma edge.

The paper is organized as follows. The ECEI diagnostic is described in more detail in Section 2. Section 3 explains the forward model that is now routinely used for the ECE Imaging at ASDEX Upgrade. Measurements of the temperature fluctuations and the mode analysis by the ECEI is presented in Section 4 . Section 5 treats the spatial localization of the mode. Forward modelling of the electron cyclotron radiation with density fluctuations in the steep gradient region is described in Section 6. In Section 7 we compare measured mode velocities with the $E \times B$ velocity. A summary is given in Section 8.

\section{ECEI at ASDEX Upgrade}

The ECEI diagnostic at ASDEX Upgrade is well suited for measurements of relative temperature fluctuations with two separate arrays looking at two toroidally separated locations inside the plasma under slightly different toroidal angles[4]. Its poloidal extension covers the regions above, across, and below the midplane. The geometry of the ECEI system, configured for the shot \#33616, is shown in figure 1. Figure 1 (a) shows the $q=6$ flux surface in toroidal geometry as the grey transparent surface. The $\mathrm{q}=6$ magnetic field line is shown as a white solid line on top of the $\mathrm{q}=6$ flux surface. The two red regions are showing the two ECEI systems and its toroidal location. Toroidal separation of two ECEI systems is about $\Delta \phi$ of $10^{\circ}$. Zoomed, the position of the ECEI arrays relative to 1D ECE radiometer is shown in figure 1(b) in Cartesian coordinates. The toroidal separation of $10^{\circ}$ from figure 1 (a) translates into $40 \mathrm{~cm}$ in Cartesian coordinates. The black circle $(\bullet)$ corresponds to the location of the ECEI channel used for the comparison with the magnetic measurements. Figure 1(c) shows the poloidal cross section of ASDEX Upgrade indicating the measurement positions for the different edge diagnostics used in this work: ECE Imaging, Li Beam Emission Diagnostic (Li-BES) 23], channels of the 1D ECE radiometer 17] and magnetic pick-up

coils (note that the $\mathrm{R}$ is defined ad $R=\sqrt{x^{2}+y^{2}}$ ). The ECE and ECEI are temperature diagnostics, the Li-BES is an edge density profile diagnostic, and the magnetic pick-up coil measure magnetic fluctuations. The ECEI, ECE and Li-BES are positioned in the same sector of ASDEX Upgrade, measuring at very close toroidal locations. The ECEI has a toroidal observation angle. When focused at the edge, array 1 has a launching toroidal angle of 7 degrees and array 2 has an angle of 5.7 degrees with respect to the the 1D ECE. As a consequence of the toroidal observation angle, the Doppler broadening of the emission lines is increased, and therefore contributes to the radial resolution of the diagnostic. In this work we use the data from the array 2 with the IF bandwidth of $390 \mathrm{MHz}$.

Emission of optically thick Maxwellian plasmas, when observed perpendicularly to 
a)

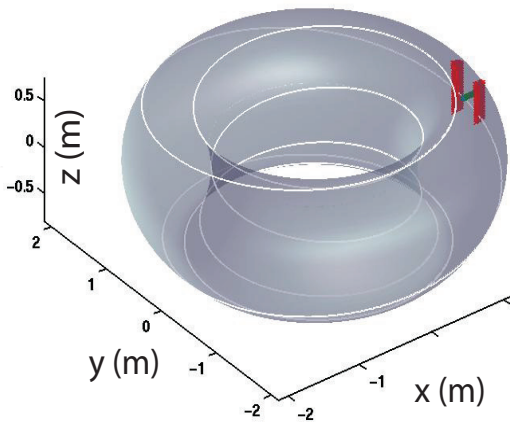

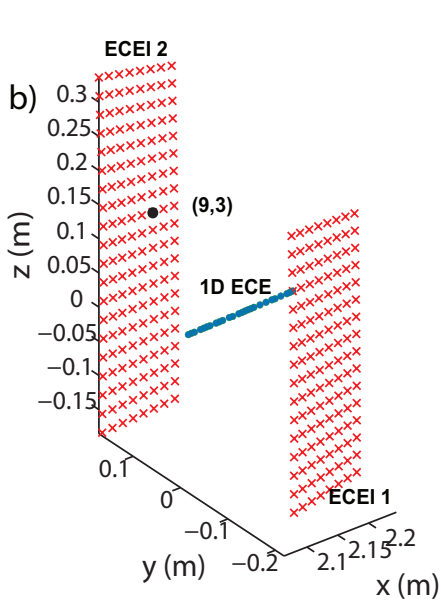

\#33616@7.2 s

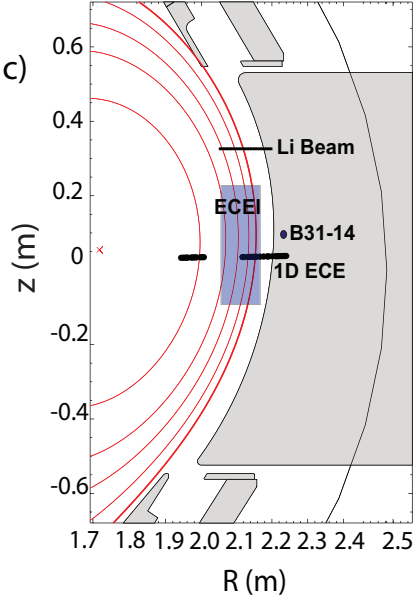

Figure 1: a) $q=6$ flux surface plotted as a gray shaded area for the shot \# 33616 at t 7.2 s. Magnetic field line for this equilibrium is plotted as white solid line. The red surfaces, intersecting the $\mathrm{q}=6$ flux tube correspond to two ECEI imaging systems. b) Absolute measurement positions of two toroidally separated ECEI arrays in Cartesian coordinates are shown as red crosses (X); edge channels of conventional ECE radiometer are shown as green dots. The ECEI channel of the ECEI 2 array that is used for comparison with the magnetic measurements is marked as black circle $(\bullet)$. c) Poloidal cross section of ASDEX Upgrade indicating the measurement positions of: the Lithium beam diagnostic, ECE Imaging, standard ECE radiometer and magnetic pick-up coil (B31-14).

the magnetic field, originates from a radially thin layer as is the case in the standard ECE diagnostic on ASDEX Upgrade. Therefore, for perpendicular propagation, the radial resolution of the system can be well approximated by the IF bandwidth of the system. ECE Imaging has a contribution of Doppler broadened emission on top of the IF broadening. When observing high density H-mode plasmas under the oblique angle, the LOS can be approximated by a straight line for lower density cases, or curved due to the high density gradient. Refraction of the beams causes an ambiguity in the measurement positions. This is of special importance for the channels at the top and the bottom of the ECEI arrays, since they encounter the strong gradients under the largest angle. Also, due to the high temperature of the plasmas, relativistic effects cannot be neglected. Because of the relativistic mass increase of the resonant electrons, the frequency will be downshifted. When combined with the low optical depth at the plasma edge, these effects make the interpretation and the origin of the electron cyclotron radiation complicated and an extended Electron Cyclotron Forward Model (ECFM), including the radiation transport effects, needs to be used as a standard tool for determining the origin of the emitted radiation [6]. 


\section{Forward modelling of the radiation detected by the ECEI}

In order to get an idea of the diagnostic constraints and characteristics, we describe the forward modelling of the electron cyclotron radiation for an H-mode discharge where we calculate the expected radiation temperatures for a given equilibrium and electron density and temperature profiles. It is important to note that the IF bandwidth is included in this modelling, the beam is approximated with a single ray and possible influence by the O-mode emission is neglected.

For given $\mathrm{T}_{e}$ and $\mathrm{n}_{e}$ profiles, ray tracing, that accounts for refraction effects, is performed for each channel and then the radiation transport equation is solved along the line of sight until it reaches back to the antenna. The input electron density and temperature profiles are shown in figure 2(a). Ray tracing, with an example of 111.6 $\mathrm{GHz}$ channels for all 20 lines of sight, is shown in figure 2(b) alongside the CLISTE equilibrium reconstruction[2]. The separatrix position is shown as a thick solid black line, whilst rays are shown as red dashed lines.

The characteristics of the emitted radiation as calculated by the ECFM of a single ECEI $(111.6 \mathrm{kHz})$ channel at the midplane are given in figure 3 . We distinguish two positions describing a single channel. The cold resonance position is only dependent on the magnetic field. It is shown as a black dashed line. When radiation transport effects are taken into account the emission position changes thus corresponding to a warm resonance position. The warm resonance is given by the peak of the birth-place distribution function $\mathrm{D}_{\omega}$, depicted as a blue solid line. Red dashed line corresponds to the peak of $\mathrm{D}_{\omega}$ for this channel. Its resolution is determined by the width of the $\mathrm{D}_{\omega}$ and would correspond to the radial extent of the plasma mostly contributing to the emitted radiation.
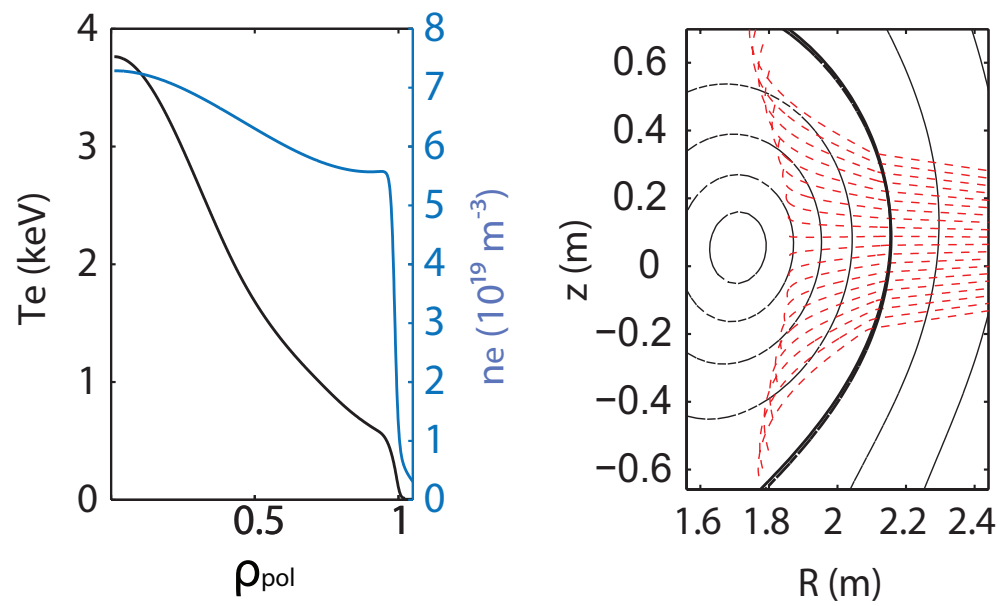

Figure 2: a) $T_{e}$ and $n_{e}$ profiles used to forward model the radiation transport. b) Ray tracing representing the lines of sight for 20 ECEI poloidally distributed channels.

The radiation transport equation is solved for every channel of one ECEI array. The two dimensional distribution of the cold and the warm resonances is shown in 
figure 4(a). It is observed that the inner most channels are Doppler shifted towards the larger major radius $\mathrm{R}$, therefore lower toroidal magnetic field, whilst the outer most channels (with the cold resonances outside the separatrix) are measuring the signal from the region inside the separatrix. This emission corresponds to the relativistically down shifted radiation. Expected radiation temperatures for ECEI channel mapped onto cold and warm resonance positions are shown in figure 4(b) and 4(c), respectively. It is observed that the measured surface area of the ECEI window, when mapped on the warm resonances, is smaller than when mapped onto a cold resonances, due to the shifts mentioned above. In the SOL region only shine-through emission from the pedestal region can be observed in the steady state conditions.

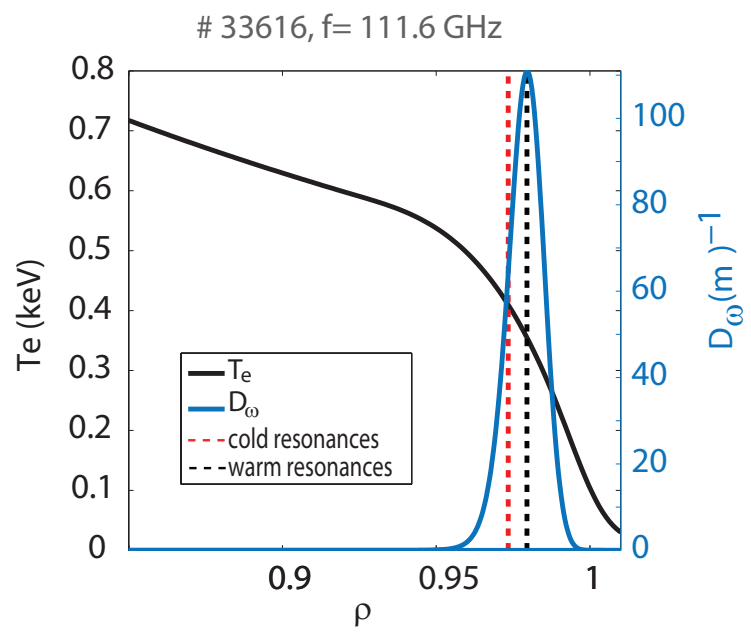

Figure 3: Origin of the observed emission (warm resonance) defined by the emitted intensity $\mathrm{D}_{\omega}$ for the $111.6 \mathrm{GHz}$ ECEI channel, at the midplane. Corresponding input $\mathrm{T}_{e}$ profile is shown as a black solid line. Warm resonance position is shown as black dashed line and corresponds to a peak of $D_{\omega}$. The cold resonance is labelled as red dashed line and depends only on the magnetic field strength. The radial width of the plasma contributing to the measured signal is determined by the width (FWHM) of the $\mathrm{D}_{\omega}$.

The above analysis shows the effect of steady state plasma conditions on the ECEI measurements. From this, one can conclude that measurements in the SOL region are not feasible with the ECEI diagnostics during steady state, and all the signal measured by channels in the SOL comes from the pedestal top. However, the presence of transient events, such as filaments, affects the location of the emitted radiation locally, as shown in figure 5 .

In order to examine the effect of filaments, we use the electron temperature and density distributions, instead of profiles. As the most realistic scenario we chose the distributions from a non-linear JOREK simulation [8] during an ELM crash. It is a simulation with two fluid effects as described in [16], with a single temperature assuming that $\mathrm{T}=\mathrm{T}_{e}=\mathrm{T}_{i}$. However, the JOREK distributions are scaled down uniformly for 


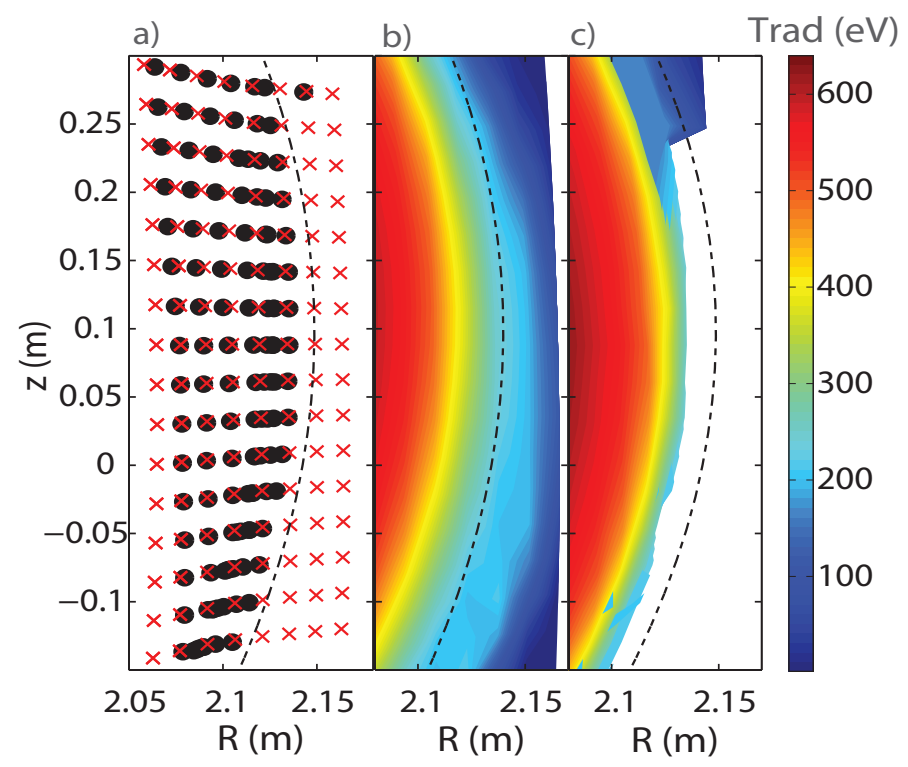

Figure 4: Shot \# 33616 at 7.5 s. a) Calculated cold resonances are labelled as red crosses $(\mathrm{X})$ and the warm resonances as black circles $(\bullet)$. The radiation temperature mapped onto positions of the cold resonances (b) and the warm resonances (c). The separatrix position is marked by a black dashed line.

about $20 \%$ in order to match with the core electron density and the temperature values at the midplane of the shot \# 33195 at time $t=2.5872$ s. In this 'hybrid' simulation the steady state equilibrium for shot \# 33195 together with the electron density and the temperature distributions are used as an input for the forward model.

As shown in figure 5(a) $n_{e}$ perturbation abruptly is crossing the separatrix, whilst $T_{e}$ shown in figure 5(b) is smooth due to fast parallel heat losses on open field lines.

Modelled cold and warm resonances for these input distributions are shown in figure 5(c) and 5(d), respectively. In figure 5(c) cold and warm resonances are plotted over the input electron density distribution. As seen, the positions of the outermost measurements match with the positions of the filaments. These outermost warm resonances of the LOSs at the midplane are in the SOL region. This shows that the presence of perturbation influences the measurement position locally shifting them towards the cold resonances. In figure 5(d), resonant positions are shown together with calculated radiation temperature. A shine-through radiation of about $300 \mathrm{eV}$ is observed in the SOL region. This shine-through originates from the filamentary structures crossing the separatrix. Although, as shown, it is possible to probe the SOL in non steady state conditions, both $\mathrm{T}_{e}$ and a shine-through is expected in this region. Thus, it is impossible to correctly interpret the measured signal without the knowledge of the local density fluctuations. 

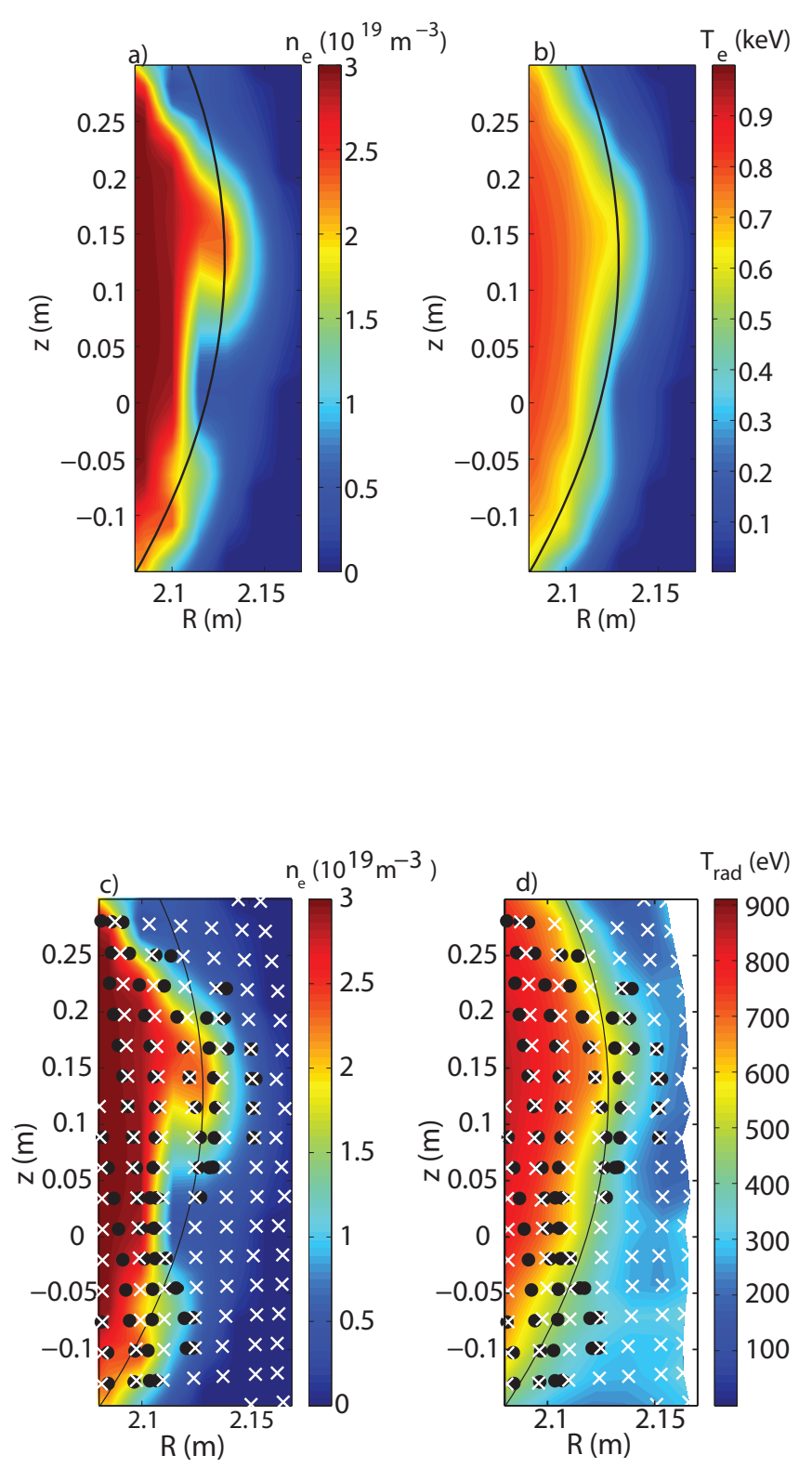

Figure 5: 2D JOREK profiles of a) Electron density; b) electron temperature. These profiles are matched to the values of the density and the temperature for the shot \# 33195 at time $\mathrm{t}=2.5872 \mathrm{~s}$ and used as an input for electron cyclotron forward model (ECFM). c) Warm resonance positions as calculated with the ECFM are marked as black circles $(\bullet)$ and the cold resonances labeled as white crosses (X) are mapped onto two dimensional distribution of the input density profile that is color codded. d) Warm resonance positions as calculated with the $\operatorname{ECFM}(\bullet)$ and the cold resonances $(\mathrm{X})$ are mapped onto a radiation temperatures obtained with the forward model for the input electron density and temperature profiles shown in a) and b), respectively. 


\section{Observations of the modes between ELM crashes}

Less violent transient events related to an ELM are inter-ELM modes. They play a crucial role in understanding an ELM cycle and may also be responsible for transport across the pedestal. So far, extensive research on this topic has been reported characterising the dynamics of the temperature fluctuations during the inter-ELM period[5, 1] with ECEI. Magnetic signature also reveal high frequency fluctuations with well defined mode numbers. They are located in the steep gradient region[15] corresponding to the location of the minimum of the edge radial electric field[12]. Evidence of the quasi-coherent mode during an inter-ELM period have been reported in[7].

In order to obtain an ECEI signal capturing ELM and inter-ELM associated phenomena, measurements are conducted during H-mode plasmas, where steep density and temperature profiles are formed (pedestal). However, to obtain reliable measurements, the cut-off density limit is avoided by focusing on moderate pedestal top density discharges. At the same time the auxiliary heating power is adjusted to obtain ELM frequencies below $100 \mathrm{~Hz}$, so that the inter-ELM dynamics of the temperature fluctuations can be measured.

From the analysis of the previous section, using the typical H-mode profiles shown in figure 2(a), we conclude that radiation temperature equals the electron temperature $T_{r}=T_{e}$ and the ECEI system delivers the information on the electron temperature $T_{e}$ and its fluctuations inside the separatrix. In this work we will use the the relative change of the electron temperature $\delta T_{e} /\left\langle T_{e}\right\rangle$ with respect to the mean value. Here, $\delta \mathrm{T}_{e}$ is defined as the $T_{e}-\left\langle T_{e}\right\rangle$, and $\left\langle T_{e}\right\rangle$ is the time average. An example of the temporal evolution of $\delta T_{r} /\left\langle T_{r}\right\rangle$ together with the divertor current as ELM indicator in the time period of $60 \mathrm{~ms}$ of the discharge \# 33616 is given in figure 6(a) and 6(b). Spectrograms of the ECEI signal and the signal measured with the magnetic pick-up coil B31-14, are shown in 6(c) and 6(d), respectively. The discharge was performed at a plasma current $\mathrm{I}_{p}=800 \mathrm{kA}$, toroidal magnetic field $\mathrm{B}_{t}=-2.52 \mathrm{~T}$ (negative sign means the opposite to $I_{p}$ ), core line averaged density $\mathrm{n}_{e}=7 \times 10^{19} \mathrm{~m}^{-3}$ and with upper triangularity $\delta_{u}=$ 0.128 and the pedestal top collisionality $\nu_{\text {ped }}^{*} \approx 1.05$.

Figure 6(a) shows the temporal evolution of the divertor shunt current that consists of combined contributions of thermoelectric and Pfirsch-Schlüter current[10]. The bursts in the divertor current are correlated with the drop in $\delta \mathrm{T}_{r} /\left\langle T_{r}\right\rangle$ measured with the ECEI channel, shown in 6(b). Recovery of the temperature fluctuations after an ELM crash happens in two steps: fast increase followed by the steady state phase. At the onset of the steady phase, a low frequency $(\sim 8 \mathrm{kHz})$ narrow band mode sets in, see figure 6(c). The duration of the mode is about $10 \mathrm{~ms}$ and vanishes just at the onset of an ELM crash. This low frequency mode correlates well in time with the high frequency modes appearing in the magnetic measurements shown in figure 6(d). Both low and the high frequency modes occur after the recovery of the maximum pressure gradient as shown in figure 6(e). In figure 6(e) we shot the temporal evolution of the pressure 


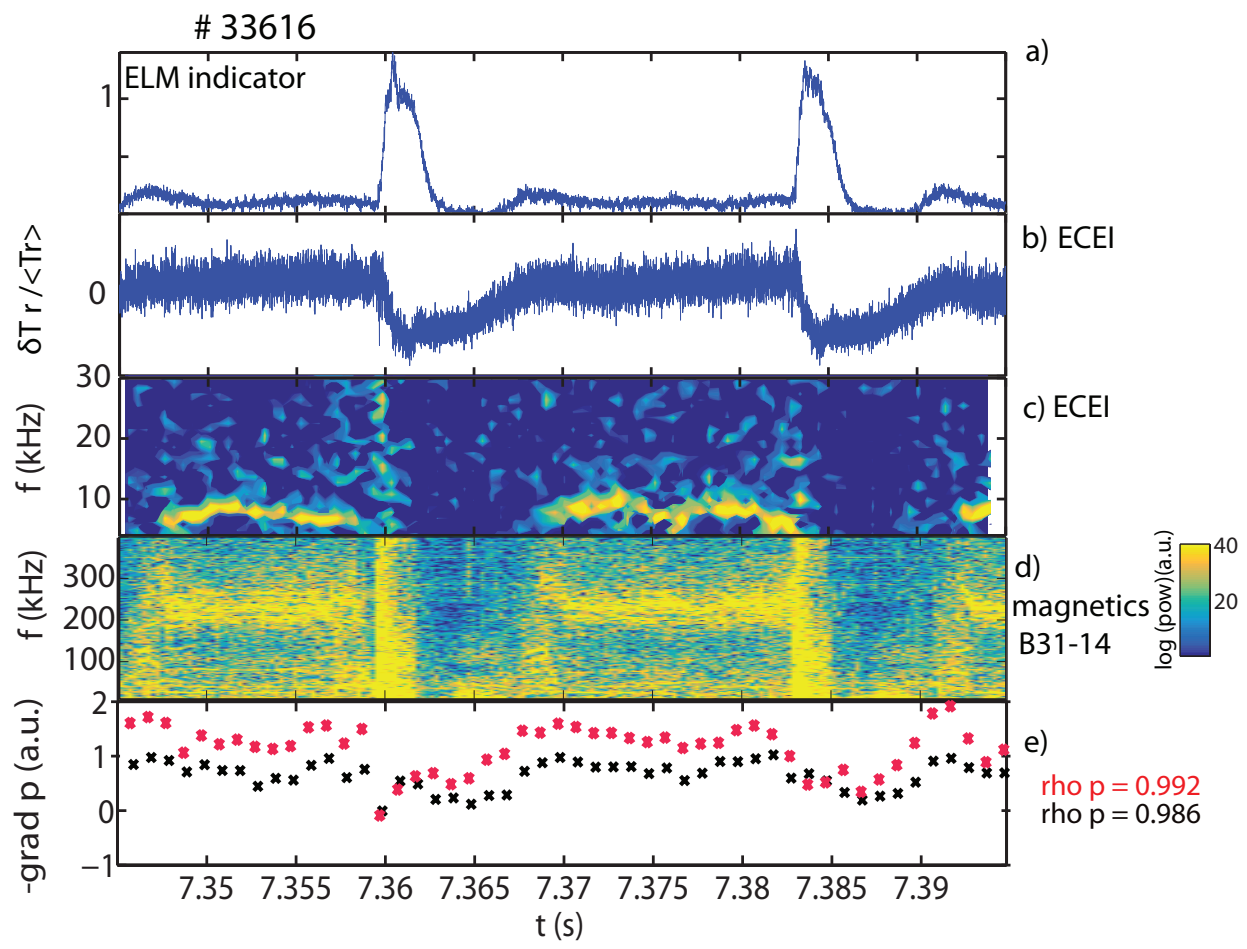

Figure 6: Shot \# 33616. Temporal evolution of the: a) Divertor current. b) Relative change of the radiation temperature $\left(\delta \mathrm{T}_{r} /\left\langle T_{r}\right\rangle\right)$ measured by the single ECEI channel in the steep gradient region. Power spectral density comparison between: c) ECEI channel; d) magnetic pick-up coil; e) pressure gradient at two nearby radial locations in the steep gradient region. The position of both ECEI channel and the magnetic coil are indicated in figure 1 .

gradient at the two nearby location in the region of steepest gradients. Correlation of the clamping of the pressure gradient and the onset of the high frequency magnetic fluctuations has been studied in [12]. The toroidal mode numbers of the high $f$ modes, determined from the magnetics are $-8,-9,-10$ showing that there are multiple modes present in this frequency region. The negative sign corresponds to the rotation of the mode in the electron diamagnetic direction.

A closer look at the inter-ELM mode is taken in figure 7. The measurement positions of the edge ECEI channels are shown in figure 7(a). All channels distributed along the flux surface, marked as red crosses see the $\sim 8 \mathrm{kHz}$ mode that modulates the relative temperature level up to $10 \%-15 \%$.

The temporal evolution of $\delta \mathrm{T}_{r} /\left\langle T_{r}\right\rangle$ measured by the single ECEI channel at the vertical position $\mathrm{z}=0.1 \mathrm{~m}$, corresponding to the magnetic midplane, is shown in figure 7 (b). A spectrogram of the midplane measurement, presented in figure 7 (c), shows the strong mode in the $\sim 8 \mathrm{kHz}$ range. The observed mode slightly changes in frequency during its lifetime. The duration of the mode is $\sim 10 \mathrm{~ms}$ and $\delta \mathrm{T}_{r} /\left\langle T_{r}\right\rangle$ during its lifetime is about $10 \%$.

Poloidally resolved measurements $\delta \mathrm{T}_{r} /\left\langle T_{r}\right\rangle$ expressed in percentage along the flux 


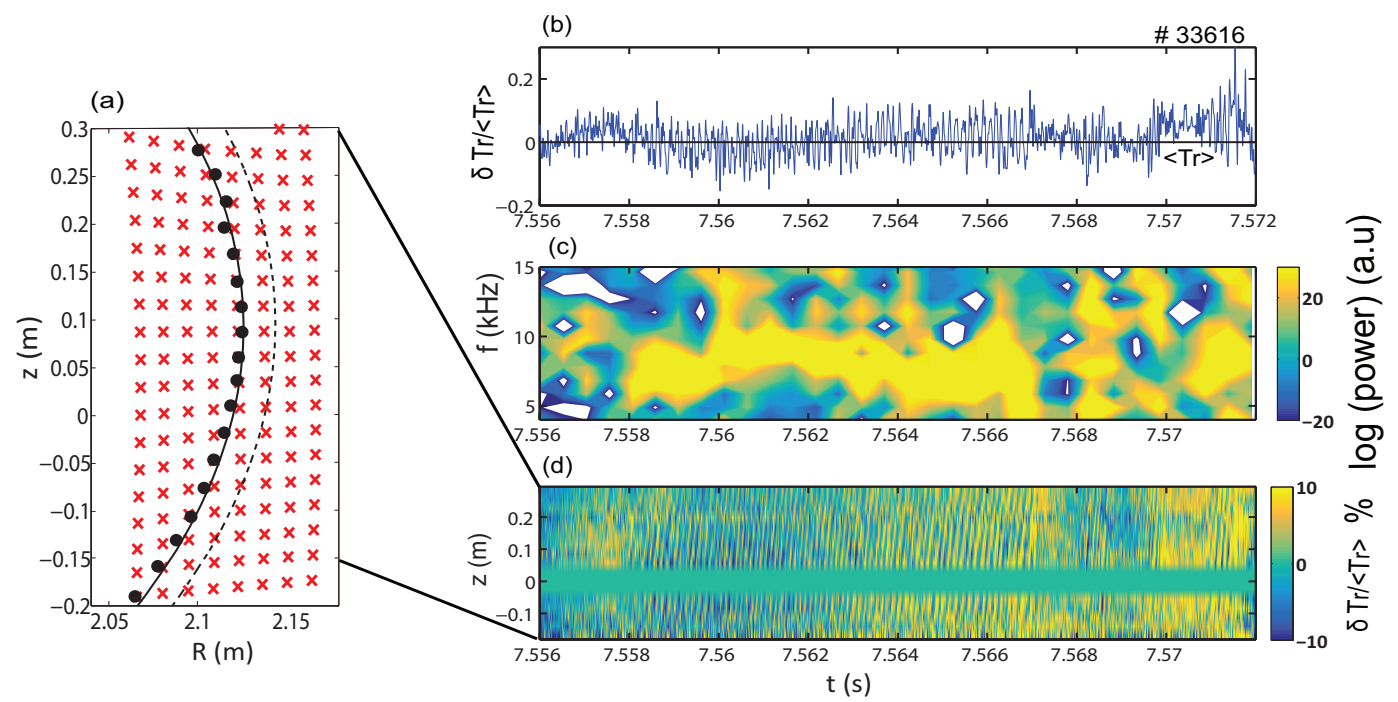

Figure 7: Localization and the temporal evolution of $\delta \mathrm{T}_{r} /\left\langle T_{r}\right\rangle$ seen in the ECEI observation window. a) Warm resonant channels $(\bullet)$ of array 2 , that measure the modulation of the radiation temperature, are distributed along the flux surface at $\rho_{\text {pol }} \sim$ 0.985. (b) Relative temperature change of a single channel $10 \mathrm{~cm}$ above the midplane. c) Time resolved spectrogram of the channel as in (b) showing the presence of the strong mode in the $7-8 \mathrm{kHz}$ range. d) Temporal evolution of $\delta \mathrm{T}_{r} /\left\langle T_{r}\right\rangle$ in percentage measured by the channels distributed along the flux surface with positions shown in (a) as black bullets. This representation visualize the propagation of $\delta \mathrm{T}_{r} /\left\langle T_{r}\right\rangle$ in the poloidal direction, from the bottom to the top channels, along the flux surface. Three channel at the midplane $(\mathrm{z}=0)$ are set to zero.

surface are shown in figure $7(\mathrm{~d})$ in a form of vertically distributed time traces. This kind of visualisation helps to follow the propagation direction of the mode. From here it can be seen that the mode propagates from the bottom to the top, corresponding to the electron diamagnetic direction.

Such poloidally resolved measurements enable the determination of the poloidal velocity of the observed mode. Figure 8 (a) is a zoom into the phase of constant frequency of the mode. Three channel at the midplane $(z=0)$ are set to zero. The quality of data for those channels was too poor due to electronics, therefore they are omitted for better graphical representation.

In order to determine the velocity of the mode in the vertical plane we use the cross correlation between a reference channel and all the other poloidally distributed channels along the flux surface as shown in figure 8(b). The velocity measured this way, shows no variations along the flux surface, and follows the straight line as indicated by the black arrow. The calculated velocity of the mode is $\sim 3 \mathrm{~km} / \mathrm{s}$ moving in the electron diamagnetic direction. Cross correlation between three vertical positions indicates the structure with three maxima fitting in the ECEI observation window. The local poloidal wavelength of this mode, as measured on the low field side, therefore is $\lambda_{\text {pol }} \sim 15 \mathrm{~cm}$. 

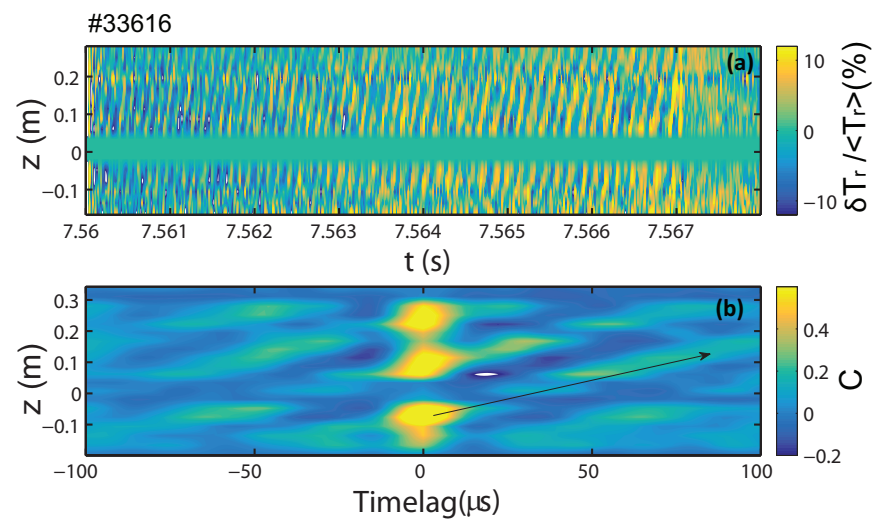

Figure 8: a) Vertically resolved $\delta \mathrm{T}_{r} /\left\langle T_{r}\right\rangle$ along the flux surface during the $8 \mathrm{kHz}$ mode duration. b) Cross-correlation of the reference channel taken at the vertical position $\mathrm{z}$ $=-0.1 \mathrm{~m}$ with all other poloidal channels. The black arrow approximates the direction of the mode propagation in space and time.

\section{Spatial localization}

To complement the analysis of the modes observed with the ECEI, data is compared with other edge diagnostics shown in figure 1(a). The Li-BES diagnostic that resolves the profiles of the electron density and its fluctuations at the plasma edge also observed the mode activity. It is also measured by the conventional 1D ECE radiometer. Comparison between conventional ECE, ECE Imaging and Li-BES measurements at the edge is shown in figure 9 in the form of spectrograms. All spectrograms show activity in the $\sim 8 \mathrm{kHz}$ range indicating the mode is present in both density and temperature measurements.

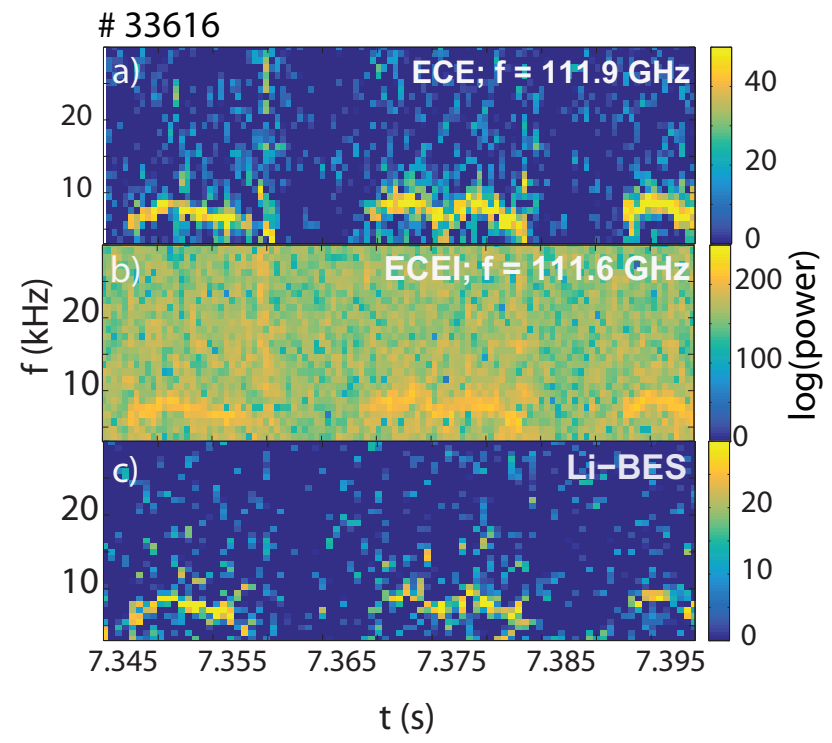

Figure 9: Power spectral density showing a narrow band mode; a) in the ECE channel, b) ECEI, c) Li beam channel 
The amplitude of the density fluctuations is estimated from the Li-BES forward model [23] in the following fashion: In order to obtain the information on the relative change $\delta n / n$ of the electron density, this quantity is varied in order to match measured fluctuations in the collected light $\delta I / I$ of $10 \%$. The deviation is assumed to be Gaussian in space, of the width that matches the radial resolution of the Li-BES system $(5 \mathrm{~mm})$. The location of the perturbation (center of the Gaussian) matches the channel where the mode is detected. The variation in the density is calculated to be between $20 \%-40$ $\%$. The background electron density at the position of the mode is between $(4-5) \times 10^{19}$ $\mathrm{m}^{-3}$.

The Li-BES and 1D ECE radial resolution of $5 \mathrm{~mm}$ allows for more accurate localisation of the mode than the one from the ECEI. The 1D ECE channel at the same location as the Li-BES channel measures the mode activity, therefore the position of the measurement corresponds to the position of its respective channels at $\rho_{\text {pol }}=0.983$. At the separatrix position at $\rho_{\text {pol }}=1$, the mode is not observed, whilst at the location $\rho_{\text {pol }}=0.97$ and inwards, ECE and ECEI do not observe the mode. Li-BES diagnostic is not sensitive in this region.

This information is further used in the forward model of the electron cyclotron emission in order to qualitatively investigate the influence of the density on the signal measured by the ECEI in the steep gradient region where the mode is measured.

\section{Forward model of the EC radiation with density fluctuations}

Following the result of the analysis of the Li-BES data, the variation in the density level is taken to be between $20 \%$ and $40 \%$. Those limits are used for the electron cyclotron forward model in order to study the radiation temperature response to the changes in the density.
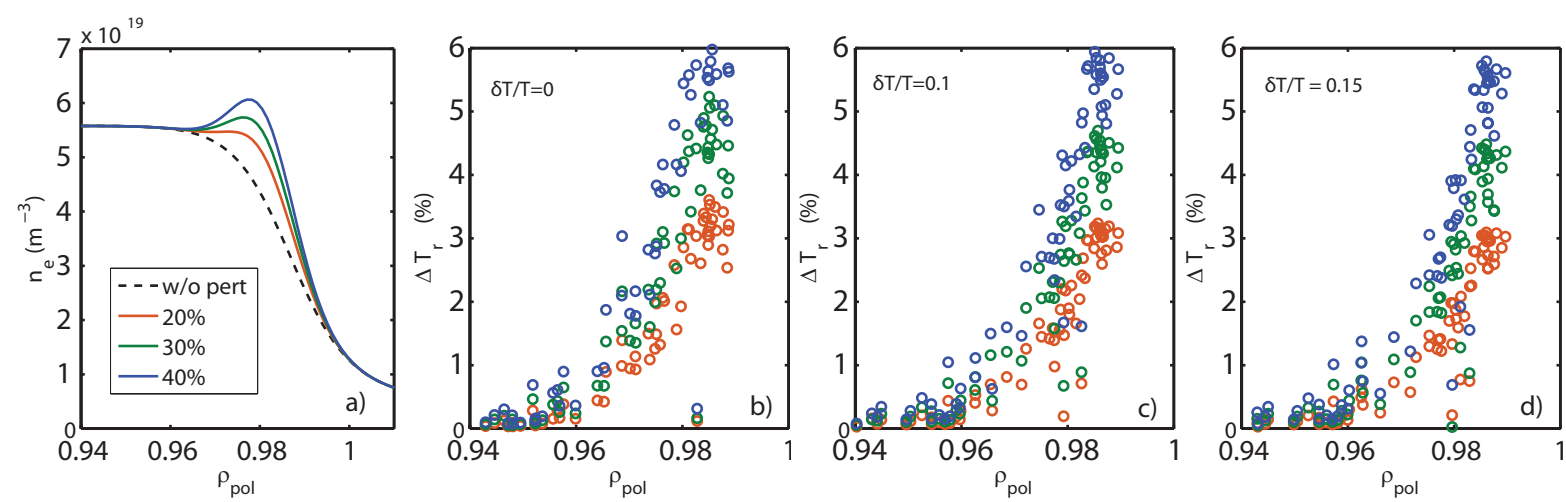

Figure 10: Radiation temperatures $\left(\Delta T_{r}\right)$ in percentage defined as $\Delta T_{r}=\delta T_{r} /\left\langle T_{r}\right\rangle$ obtained with the ECFM for the different levels of the density variations as shown in a). b) Radiation temperatures without input temperature variation. c) with input temperature variation of $10 \%$. d) with the input temperature variation of $15 \%$. 
In order to model the response of the radiation temperature to the density change, the same steps are applied as explained in Section 3. This study requires the input profiles with inserted density perturbation that mimics the one measured by the LiBES diagnostic. The shape of the perturbation is the same as the one used in the Li-BES forward model.

Modelling accounts for three different scenarios assuming that we have the cases where the temperature and the density are in phase but of different amplitudes. For this reason, the $\delta T / T$ of to $0,0.1,0.15$ is added on top of the background $T e_{e}$ profile. For each case we varied the density profile as shown in figure 10.

Figure 10 (a) shows the input density profiles used for the modelling. The density variation is between $20-40 \%$ and is color-coded.

Variations in the radiation temperatures cannot be distinguished between the three cases for $\delta T_{e} / T_{e}$ of $0,0.1,0.15$ shown in figure 10(a), 10(b), 10(c), respectively. The variation in the density $\left(\delta n_{e} / n_{e}\right)$ for each case induces the change of the radiation temperature $\Delta T_{r}$. At the location of the mode $\left(\rho_{p o l}=0.983\right)$ we can see that $20 \%$ of the density perturbation is captured in the radiation temperatures inducing $3 \%$ change in the radiation temperature. Change in the $T_{r}$ increases with the amplitude of the density variation. It is important to note that in the forward model we omitted the possible O-mode contribution and that the complex ECEI beams are approximated with a single array. This already simplifies the complexity of the ECEI geometry, therefore the modelled and measured amplitudes cannot be directly compared.

\section{Mode velocities and comparison to the $\mathbf{v}_{E \times B}$ velocity}

The mode velocity in the ECEI reference frame is $v_{\text {mode }}=v_{E \times B}+v_{p h}$, where $v_{E \times B}$ is governed by the radial electric field $E_{r}$, and $v_{p h}$ is the intrinsic phase velocity of the mode. The $E_{r}$ profile for this discharge is estimated using the neoclassical approximation for the poloidal flow of impurities [20], using experimental profiles of $n_{\mathrm{e}}$ and $T_{i}$. The $n_{\mathrm{e}}$ and reconstructed $E_{\mathrm{r}}$ profiles are shown in figure 11(a). The position of the highest perturbation in the signal, detected with different diagnostics, is within the color coded areas and those areas represent the radial extent of the plasma contributing to this measurement. Grey corresponds to the radial resolution of the ECEI and red to the Li-BES and ECE, respectively. It can be seen that the ECEI averages the signal over a much wider range of plasma radius. If we assume that he location of the mode is where the perturbation in the diagnostic measurements peaks, the radial resolution of the LiBES and ECE then enable localization of the mode to a precision of $5 \mathrm{~mm}$ as explained

in the Section 5. The background $v_{E \times B}$ velocity is calculated as $v_{E \times B}(r)=\frac{E \overrightarrow{(r)} \times B(\vec{r})}{B(r)^{2}}$ and is shown in figure 11 (b). The evaluated $\mathrm{v}_{E \times B}$ at the position of the perturbation peak is about $25 \mathrm{~km} / \mathrm{s}$ and matches with the minimum of the $E_{\mathrm{r}}$. This is, however, not in agreement with the velocity obtained from the poloidaly distributed ECEI channels, which is measured to be $3 \mathrm{~km} / \mathrm{s}$.

The high frequency modes (see figure 6(d)) are observed at the same time as the 

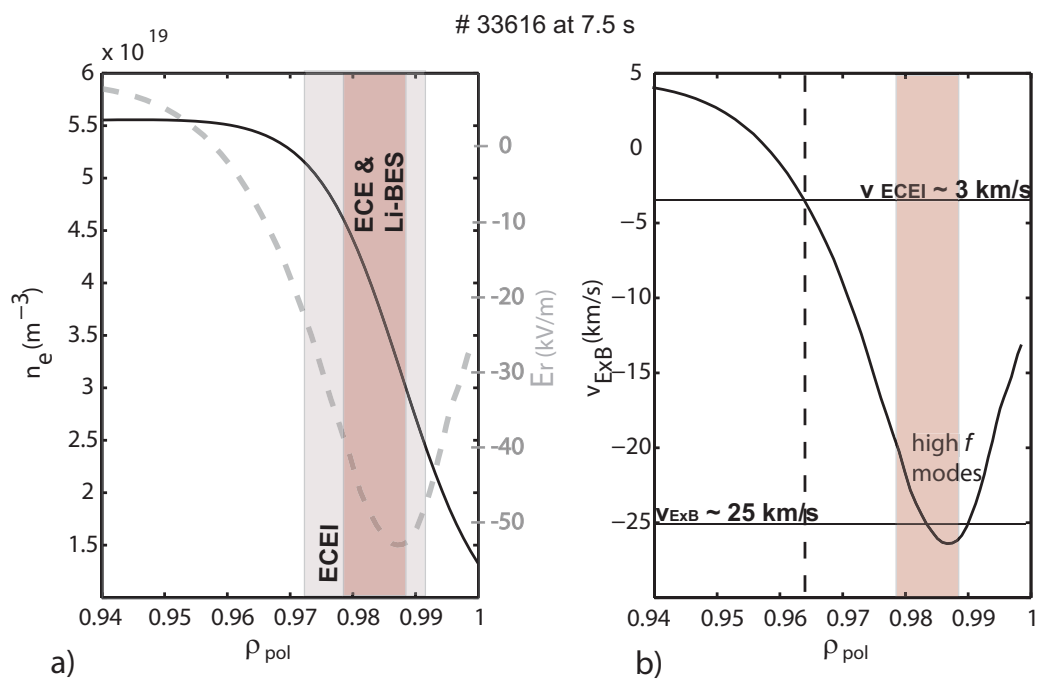

Figure 11: Shot \# 33616 at $\mathrm{t}=7.5$ s. a) Density profile of the steep gradient edge region shown as a black solid line; radial electric field $E_{r}$ estimated from the poloidal flow of impurities is presented as a dashed gray line. Radial resolution of the edge diagnostics used in this work is color coded: red corresponding to the Li-BES and 1D ECE channel; grey corresponding to the ECEI channel. b) $E \times B$ velocity calculated using the $E_{r}$. The vertical dashed line shows the position of the mode in case the velocity of the mode would match exactly the $E \times B$ velocity.

low frequency modes on the magnetic pick-up coils measuring at the low field side. As shown in [12] the high frequency modes measured by the pick-up coils are located in the minimum of the $E_{\mathrm{r}}$. From the measured frequency of the high frequency modes, if the mode is located on the rational surface $q=\frac{m}{n}$, one can determine its poloidal velocity as the $v_{\text {mode }}=2 \cdot \pi \cdot r \frac{f}{q \cdot n}$, where $r$ is the radial location of the mode and $f$ its frequency. Using the following parameters of $q=5, f=240 \mathrm{kHz}, n=8$ and $r=0.6 \mathrm{~m}$, the calculated mode velocity is $\sim 22 \mathrm{~km} / \mathrm{s}$ and is comparable with the $E \times B$ velocity within the measurement uncertainties. The propagation direction of both low and the high frequency modes is in the electron diamagnetic direction and their velocities differ by almost a factor of 10 .

However, the maximum of the perturbation does not necessarily have to coincide with the position of the mode. The mode located at the pedestal top could cause the displacement in the region of the steepest gradients where the diagnostics are most sensitive. If the mode is located at the pedestal top, the velocity of the mode would match the $\mathrm{v}_{E \times B}$ velocity within the measurements uncertainty. This would correspond to a $\rho_{\text {pol }}$ of about 0.964 , marked as the vertical dashed black line shown in figure $11(\mathrm{~b})$. The background density at this position is $5.5 \times 10^{19} \mathrm{~m}^{-3}$. Thus low sensitivity of the diagnostics at the pedestal top could cause the ambiguity in the measurement position. This effect will be further investigated. 


\section{Summary and Discussion}

In summary, it is shown that SOL measurements with the ECEI are not feasible in steady state due to the shine-through emission in this region. The radiation temperature in the SOL region, which is a region of low optical depth, is down-shifted emission from the electrons in the tail of the Maxwellian originating in the pedestal region.

However, the presence of ELM filaments in the SOL region can change the optical depth locally, due to the locally higher density and temperature, therefore both, shinethrough as well as the local emission can be observed simultaneously. In this case it is difficult to distinguish between the two contributions without the local density measurements.

In the $\mathrm{H}$-mode discharge examined in this work, a strong $8 \mathrm{kHz}$ mode has been observed with different edge diagnostics between ELMs. The mode is highly correlated with the high frequency $(\sim 200 \mathrm{kHz})$ inter-ELM modes detected on the magnetic pickup coils since the both branches set in the phase of the clamped pressure gradient before an ELM crash. Another interesting feature is that the high frequency modes are localized at the position where the diagnostics measure low frequency amplitude modulation of $8 \mathrm{kHz}$. This position matches with the minimum of the radial electric field and the propagation direction of both low and the high frequency modes is in the electron diamagnetic direction, in agreement with the sign of the $\mathrm{E}_{r}$. The magnetic

pick-up coils measure multiple modes with well defined toroidal mode numbers whilst the ECEI/ECE/Li-BES measures a single mode at low frequency. The low frequency mode is also seen on the magnetics, but of very low amplitude and its mode numbers remained undetermined.

The cross-correlation analysis from the ECEI measurements has shown the poloidal structure of the low frequency mode with the local poloidal wavelength $\lambda_{p}=15 \mathrm{~cm}$. The poloidal mode velocity as calculated from the signals of the ECEI channels distributed along the flux surface is $3 \mathrm{~km} / \mathrm{s}$, rotating in the electron diamagnetic direction. With the high spatial resolution of the Li-BES and 1D ECE diagnostics, the peak of the perturbation in the signal amplitude is measured with higher accuracy than the one of the ECEI diagnostic. The same perturbation in between ELMs is observed in the density measurements with the Li-BES diagnostic. Since measurements of the Li-BES are sensitive to the changes in the density it is concluded that $\mathrm{n}_{e}$ fluctuations contribute to the ECEI measurements. To account for this we used forward model of the electron cyclotron emission by adding a Gaussian perturbation in the pedestal region to mimic the possible density fluctuations in that region. This study has shows that the density fluctuation level can influence the measurements of ECEI and contribute to the measured radiation temperature $T_{r}$. Thus, knowledge about the local density values is necessary for correct interpretation of the measured signal.

Taking into account the measurement facts and the ambiguities in measurements due to the diagnostics sensitivity, only the hypotheses can be proposed as possible explanation for the appearance of the low frequency mode and those will be further 
investigated.

One hypothesis supports the idea of a non linear interaction between two or more high frequency modes that as a result give the low frequency mode. This resulting mode is non linearly amplified in the low field side region where it is measured by the ECEI. Experimental findings that support this theory have already been reported on different machines. Significant modification of the mode structure due to the non-linear coupling has been reported on TCV [22] and similar amplitude modulation has been observed in KSTAR [11] and explained by a superposition of two modes with different poloidal velocities. In the case studied in this manuscript, the result of the multiple mode interaction would be the wave with a measured group velocity of $3 \mathrm{~km} / \mathrm{s}$.

Another hypothesis is that the measured mode coexist together with the high frequency modes, at the same position, but very weak in amplitude compared to the high frequency modes. In this case, the $E \times B$ velocity of $25 \mathrm{~km} / \mathrm{s}$ and the measured mode velocity of $3 \mathrm{~km} / \mathrm{s}$ would result in a mode with a large phase velocity $v_{p h}=22$ $\mathrm{km} / \mathrm{s}$, in the ion diamagnetic direction.

The third possibility is that the mode is located where the measured velocity of the mode matches the $E \times B$ velocity. In this case the position of the mode is at the pedestal top and mode has no resolvable intrinsic phase velocity $\left(v_{p h}=0\right)$. The mode has well defined structure rotating in the electron diamagnetic direction in agreement with the $E \times B$ at that location. The nature of the mode could be assigned to the density fluctuation at the pedestal top that influences the steepest gradient region, where the diagnostics is most sensitive.

To distinguish between the possible explanations on the origin of the mode in near future work we will examine the poloidal velocities of the low frequency modes with a variation of plasma parameters which are known to change the frequency of the high frequency modes, i.e. edge safety factor $q$ and the ion pressure gradient $\nabla p_{i}$. Also, we will compare the dynamics of the fluctuations measured by the ECEI with newest non-linear JOREK simulation comprising an inter-ELM period. Further investigation on the sensitivity of the measurements in the pedestal top region will be conducted. Possible effect of the low frequency mode on transport will also be assessed in the future analysis.

The ECEI diagnostic, with its excellent poloidal resolution, is a useful tool for characterizing fluctuations at the edge by means of their poloidal size and velocity. The signal, however, can be dominated by the changes in the density profile and does not necessarily reflect the amplitude of the $T_{\mathrm{e}}$ fluctuations.

\section{Acknowledgements}

The author would like to thank Thomas Hayward for his constructive criticism of the manuscript.

This work has been carried out within the framework of the EUROfusion Consortium and has received funding from the Euratom research and training 
programme 2014-2018 under grant agreement No 633053. The views and opinions expressed herein do not necessarily reflect those of the European Commission.

\section{References}

[1] J.E. Boom, I.G.J. Classen, P.C. de Vries, T. Eich, E. Wolfrum, W. Suttrop, R.P. Wenninger, A.J.H. Donné, B.J. Tobias, C.W. Domier, N.C. Luhmann Jr, H.K. Park, and the ASDEX Upgrade Team. 2D ECE measurements of type-I edge localized modes at ASDEX upgrade. Nuclear Fusion, 51(10):103039, 2011.

[2] P J Mc Carthy and ASDEX Upgrade Team. Identification of edge-localized moments of the current density profile in a tokamak equilibrium from external magnetic measurements. Plasma Physics and Controlled Fusion, 54(1):015010, 2012.

[3] I. G. J. Classen, J. E. Boom, W. Suttrop, E. Schmid, B. Tobias, C. W. Domier, N. C. Luhmann Jr., A. J. H. Donné, R. J. E. Jaspers, P. C. de Vries, H. K. Park, T. Munsat, M. Garcia-Munoz, and P. A. Schneider. 2D electron cyclotron emission imaging at ASDEX upgrade (invited). Review of Scientific Instruments, 81(10):10D929, 2010.

[4] I. G. J. Classen, C. W. Domier, N. C. Luhmann Jr., A. V. Bogomolov, W. Suttrop, J. E. Boom, B. J. Tobias, and A. J. H. Donné. Dual array 3D electron cyclotron emission imaging at ASDEX upgrade. Review of Scientific Instruments, 85(11):11D833, 2014.

[5] I.G.J. Classen, J.E. Boom, A.V. Bogomolov, E. Wolfrum, M. Maraschek, W. Suttrop, P.C. de Vries, A.J.H. Donné, B.J. Tobias, C.W. Domier, N.C. Luhmann, and the ASDEX Upgrade Team. The role of temperature fluctuations in the dynamics of type-I and type-ii edge localized modes at ASDEX upgrade. Nuclear Fusion, 53(7):073005, 2013.

[6] Denk, Severin S., Fischer, Rainer, Maj, Omar, Poli, Emanuele, Stober, Jrg K., Stroth, Ulrich, Vanovac, Branka, Suttrop, Wolfgang, Willensdorfer, Matthias, and ASDEX Upgrade Team.

Radiation transport modelling for the interpretation of oblique ece measurements. EPJ Web Conf., 147:02002, 2017.

[7] A. Diallo, R. J. Groebner, T. L. Rhodes, D. J. Battaglia, D. R. Smith, T. H. Osborne, J. M. Canik, W. Guttenfelder, and P. B. Snyder. Correlations between quasi-coherent fluctuations and the pedestal evolution during the inter-edge localized modes phase on DIII-D. Physics of Plasmas, 22(5):056111, 2015.

[8] G.T.A. Huysmans and O. Czarny. MHD stability in x-point geometry: simulation of ELMs. Nuclear Fusion, 47(7):659, 2007.

[9] M. Jiang, Z. B. Shi, S. Che, C. W. Domier, N. C. Luhmann Jr., X. Hu, A. Spear, Z. T. Liu, X. T. Ding, J. Li, W. L. Zhong, W. Chen, Y. L. Che, B. Z. Fu, Z. Y. Cui, P. Sun, Y. Liu, Q. W. Yang, and X. R. Duan. Development of electron cyclotron emission imaging system on the hl-2A tokamak. Review of Scientific Instruments, 84(11):113501, 2013.

[10] A. Kallenbach, A. Carlson, G. Pautasso, A. Peeters, U. Seidel, and H.-P. Zehrfeld. Electric currents in the scrape-off layer in asdex upgrade. Journal of Nuclear Materials, 290-293(Supplement C):639 - 643, 2001. 14th Int. Conf. on Plasma-Surface Interactions in Controlled Fusion D evices.

[11] M. Kim, J. Lee, H.K. Park, G.S. Yun, W. Lee, C.W. Domier, Luhmann N.C. Jr., and KSTAR team. Multimode excitation during the inter-ELM crash periods in KSTAR $\mathrm{h}$-mode plasma. Nuclear Fusion, 55(7):073001.

[12] F M Laggner, E Wolfrum, M Cavedon, F Mink, E Viezzer, M G Dunne, P Manz, H Doerk, G Birkenmeier, R Fischer, S Fietz, M Maraschek, M Willensdorfer, F Aumayr, the EUROfusion MST1 Team, and the ASDEX Upgrade Team. High frequency magnetic fluctuations correlated with the inter-elm pedestal evolution in ASDEX upgrade. Plasma Physics and Controlled Fusion, 58(6):065005, 2016.

[13] A. W. Leonard. Edge-localized-modes in tokamaks. Physics of Plasmas, 21(9):090501, 2014.

[14] Y R Martin, T Takizuka, and the ITPA CDBM H-mode Threshold Database Working Group. 
Power requirement for accessing the h-mode in ITER. Journal of Physics: Conference Series, 123(1):012033, 2008.

[15] Felician Mink, Elisabeth Wolfrum, Marc Maraschek, Hartmut Zohm, Lszl Horvth, Florian M Laggner, Peter Manz, Eleonora Viezzer, Ulrich Stroth, and the ASDEX Upgrade Team. Toroidal mode number determination of ELM associated phenomena on ASDEX upgrade. Plasma Physics and Controlled Fusion, 58(12):125013, 2016.

[16] F. Orain, M. Bcoulet, G. Dif-Pradalier, G. Huijsmans, S. Pamela, E. Nardon, C. Passeron, G. Latu, V. Grandgirard, A. Fil, A. Ratnani, I. Chapman, A. Kirk, A. Thornton, M. Hoelzl, and P. Cahyna. Non-linear magnetohydrodynamic modeling of plasma response to resonant magnetic perturbations. Physics of Plasmas, 20(10):102510, 2013.

[17] W. Suttrop and A.G. Peeters. Practical limitations to plasma edge ewlectron temperature measurements by radiometry of electron cyclotron emission. IPP report, 1996.

[18] B. Tobias, C. W. Domier, T. Liang, X. Kong, L. Yu, G. S. Yun, H. K. Park, I. G. J Classen, J. E. Boom, A. J. H. Donné, T. Munsat, R. Nazikian, M. Van Zeeland, R. L. Boivin, and N. C. Luhmann Jr. Commissioning of electron cyclotron emission imaging instrument on the DIII-D tokamak and first data. Review of Scientific Instruments, 81(10):10D928, 2010.

[19] B. J. Tobias, M. E. Austin, J. E. Boom, K. H. Burrell, I. G. J. Classen, C. W. Domier, N. C. Luhmann Jr., R. Nazikian, and P. B. Snyder. Ece-imaging of the h-mode pedestal (invited). Review of Scientific Instruments, 83(10):10E329, 2012.

[20] E. Viezzer, T. Pütterich, G.D. Conway, R. Dux, T. Happel, J.C. Fuchs, R.M. McDermott, F. Ryter, B. Sieglin, W. Suttrop, M. Willensdorfer, E. Wolfrum, and the ASDEX Upgrade Team. Highaccuracy characterization of the edge radial electric field at asdex upgrade. Nuclear Fusion, 53(5):053005, 2013.

[21] F. Wagner, G. Becker, K. Behringer, D. Campbell, A. Eberhagen, W. Engelhardt, G. Fussmann, O. Gehre, J. Gernhardt, G. v. Gierke, G. Haas, M. Huang, F. Karger, M. Keilhacker, O. Klüber, M. Kornherr, K. Lackner, G. Lisitano, G. G. Lister, H. M. Mayer, D. Meisel, E. R. Müller, H. Murmann, H. Niedermeyer, W. Poschenrieder, H. Rapp, H. Röhr, F. Schneider, G. Siller, E. Speth, A. Stäbler, K. H. Steuer, G. Venus, O. Vollmer, and Z. Yü. Regime of improved confinement and high beta in neutral-beam-heated divertor discharges of the ASDEX tokamak. Phys. Rev. Lett., 49:1408-1412, Nov 1982.

[22] R.P. Wenninger, H. Reimerdes, O. Sauter, and H. Zohm. Non-linear magnetic perturbations during edge-localized modes in TCV dominated by low n mode components. Nuclear Fusion, 53(11):113004, 2013.

[23] M Willensdorfer, G Birkenmeier, R Fischer, F M Laggner, E Wolfrum, G Veres, F Aumayr, D Carralero, L Guimaris, B Kurzan, and the ASDEX Upgrade Team. Characterization of the Li-BES at ASDEX upgrade. Plasma Physics and Controlled Fusion, 56(2):025008, 2014.

[24] L Yu, C W Domier, X Kong, S Che, B Tobias, H Park, C X Yu, and N C Luhmann Jr. Recent advances in ECE imaging performance. Journal of Instrumentation, 7(02):C02055, 2012.

[25] G. S. Yun, W. Lee, M. J. Choi, J. Lee, M. Kim, J. Leem, Y. Nam, G. H. Choe, H. K. Park, H. Park, D. S. Woo, K. W. Kim, C. W. Domier, N. C. Luhmann Jr., N. Ito, A. Mase, and S. G. Lee. Quasi 3D ECE imaging system for study of MHD instabilities in KSTAR. Review of Scientific Instruments, 85(11):11D820, 2014.

[26] H. Zohm. Edge localized modes (ELMs). Plasma Physics and Controlled Fusion, 38(2):105, 1996. 